\title{
A Novel Condition to the Harmonic of the Velocity Vector Field of a Curve in $R^{n}$
}

\author{
Ayşe Altın and Canan Köroğlu \\ Department of Mathematics, Faculty of Science, Hacettepe University, Beytepe, 06800 Ankara, Turkey \\ Correspondence should be addressed to Canan Köroğlu; ckoroglu@hacettepe.edu.tr
}

Received 30 March 2017; Accepted 8 May 2017; Published 8 June 2017

Academic Editor: Gisèle R. Goldstein

Copyright ( 2017 Ayșe Altın and Canan Köroğlu. This is an open access article distributed under the Creative Commons Attribution License, which permits unrestricted use, distribution, and reproduction in any medium, provided the original work is properly cited.

In this paper, a condition is obtained for the harmonic of the velocity vector field in the curve family passing through the fixed $p$ and $q$ points in $R^{n}$. It shows that the condition can be expressed in terms of the curvature functions. Finally, we give an example which provides the mentioned condition in this work and illustrates it with figures.

\section{Introduction}

Differential geometry is applied to other fields of science and mathematics. In particular, it applied various problems in mechanics, computer-aided as well as traditional engineering design, physics, geodesy, geography, space travel, and relativity theory [1].

The volume of unit vector fields has been studied by Gluck and Ziller [2], Johnson [3], and Higuchi et al. [4], among other scientists. In [5], the energy of a unit vector field $X$ on a Riemannian manifold $M$ is defined as the energy of the mapping $X: M \rightarrow T^{1} M$, where the unit tangent bundle $T^{1} M$ is equipped with the restriction of the Sasaki metric on TM.

Generally, any geometric problem about curves can be solved using the curves' Frenet vectors field. Therefore, in [6], we focus on the curve $C$ instead of the manifold $M$. For a given curve $C$, with a pair of parametric unit speeds $(I, \alpha)$ in a space $R^{n}$ we denote Frenet frames at the points $\alpha(a)$ and $\alpha(s)$ by $\left\{V_{1}(a), V_{2}(a), \ldots, V_{r}(a)\right\}$ and $\left\{V_{1}(s), V_{2}(s), \ldots, V_{r}(s)\right\}$, respectively, as we take a fixed point $a \in I$. We calculate the energy of the Frenet vectors fields and the angle between the vectors $V_{i}(a)$ and $V_{i}(s)$, where $1 \leq i \leq r$. So, we see that both energy and angle depend on the curvature functions of the curve $C$.

In this paper, we choose two points $p$ and $q$ in $R^{n}$. We obtain a condition for the harmonic of the velocity vector field in the curve family of all curves from $p$ to $q$ points. Thus, we notice that this condition can be expressed in terms of the curvature functions. Finally, we give an example which provides the mentioned condition in this work and illustrate it with figures.

Definition 1. A curve segment is the portion of a curve defined in a closed interval $[a, b][7]$.

Definition 2. Let $(I, \alpha)$ be a parametric pair for a curve $C$ in a space $R^{n}$ and $\left\{V_{1}(s), V_{2}(s), \ldots, V_{r}(s)\right\}$ be Frenet frames at the point $\alpha(s) \in C$. Let

$$
k_{i}(s)=\left\langle V_{i}^{\prime}(s), V_{i+1}(s)\right\rangle, \quad \forall s \in I, \quad 1 \leq i \leq r
$$

be defined as curvature function on $C$ and the real number $k_{i}(s)$ be defined as $i$ th curvature on $C$ at the point $\alpha(s)$.

Theorem 3 (Frenet formulas). Let $(I, \alpha)$ be a parametric pair for a curve $C$ in a space $R^{n}$. If we take ith curvature $k_{i}(s)$ and Frenet frames $\left\{V_{1}(s), V_{2}(s), \ldots, V_{r}(s)\right\}$ at the point $\alpha(s)$, then the following relations are hold:

$$
\begin{aligned}
& V_{i}^{\prime}(s)=k_{1}(s) V_{2}(s), \\
& V_{i}^{\prime}(s)=-k_{i-1}(s) V_{i-1}(s)+k_{i}(s) V_{i+1}(s), \quad 1 \leq i \leq r \\
& V_{r}^{\prime}(s)=-k_{r-1}(s) V_{r-1}(s) .
\end{aligned}
$$


Proposition 4. The connection map $K: T\left(T^{1} M\right) \rightarrow T^{1} M$ verifies the following conditions:

(1) $\pi \circ K=\pi \circ d \pi$ and $\pi \circ K=\pi \circ \tilde{\pi}$, where $\tilde{\pi}: T\left(T^{1} M\right) \rightarrow$ $T^{1} M$ is the tangent bundle projection and $\pi: T^{1} M \rightarrow$ $M$ is the bundle projection.

(2) For $\omega \in T_{x} M$ and a section $\xi: M \rightarrow T^{1} M$, we have

$$
K(d \xi(\omega))=\nabla_{\omega} \xi,
$$

where $\nabla$ is the Levi-Civita covariant derivative [8].

Definition 5. For $\eta_{1}, \eta_{1} \in T_{\xi}\left(T^{1} M\right)$ we define

$$
g_{s}\left(\eta_{1}, \eta_{1}\right)=\left\langle d \pi\left(\eta_{1}\right), d \pi\left(\eta_{2}\right)\right\rangle+\left\langle K\left(\eta_{1}\right), K\left(\eta_{2}\right)\right\rangle .
$$

This gives a Riemannian metric on TM. Recall that $g_{s}$ is called the Sasaki metric. The metric $g_{s}$ makes the projection $\pi: T^{1} M \rightarrow M$ a Riemannian submersion [8].

Definition 6. The energy of a differentiable map $f:(M, g) \rightarrow$ $(N, h)$ between Riemannian manifolds is given by

$$
\mathscr{E}(f)=\frac{1}{2} \int_{M} \sum_{a=1}^{n} h\left(d f\left(e_{a}\right), d f\left(e_{a}\right)\right) v_{g},
$$

where $v_{g}$ is the canonical volume form in $M$ and $\left\{e_{a}\right\}$ is a local basis of the tangent space $[5,9]$.

Let $C^{\infty}(M ; N)$ denote the space of all smooth maps from $M$ to $N$. A map $f: M \rightarrow N$ is said to be harmonic if it is an extremal (i.e., critical point) of the energy functional $\mathscr{E}(\cdot ; D)$ : $C^{\infty}(M ; N) \rightarrow R$ for any compact domain $D$.

By a (smooth) variation of $f$ we mean a smooth map : $M \times(-\varepsilon, \varepsilon) \rightarrow N,(x, t) \rightarrow f_{t}(x)(\varepsilon>0)$ such that $f_{0}=f$. We can think of $\left\{f_{t}\right\}$ as a family of smooth mappings which depends "smoothly" on a parameter $t \in(-\varepsilon, \varepsilon)$.

Definition 7. In [10], A smooth map $f:(M, g) \rightarrow(N, h)$ is said to be harmonic if

$$
\left.\frac{d}{d t} \mathscr{E}\left(f_{t} ; D\right)\right|_{t=0}=0
$$

for all compact domains $D$ and all smooth variations $\left\{f_{t}\right\}$ of $f$ supported in $D$, where $\mathscr{E}(f ; D)=(1 / 2) \int_{D} \sum_{a=1}^{n} h\left(d f\left(e_{a}\right)\right.$, $\left.d f\left(e_{a}\right)\right) v_{g}$.

\section{A Condition for the Curve Where the Velocity Vector Field Is Harmonic}

The following theorem characterizes a critical point of the energy of the velocity vector field of a curve in $R^{n}$.

Theorem 8. Let $\alpha$ be unit speed curve in $R^{n}$ and $\alpha(a)=p$, $\alpha(b)=q$. If the velocity vector field of $\alpha$ along from $p$ to $q$ is harmonic, then the following equation is satisfied:

$$
\int_{a}^{b} \lambda(s) k_{1}(s) k_{1}^{\prime}(s) d s=0,
$$

where $k_{1}$ is the 1 th curvature function and $\lambda$ is the real-valued function on $[a, b]$.
Proof. Let $\alpha: I \rightarrow R^{n}$ be a unit speed curve in $R^{n}$ and $[a, b] \subset$ $I, \alpha(a)=p, \alpha(b)=q$. There exists a real-valued function $\lambda$ on $[a, b], \lambda(s)=(s-a)(b-s), \lambda(a)=\lambda(b)=0$, and $\lambda(s) \neq 0$ for all $s \in(a, b)$. Let $\left\{V_{1}, V_{2}, \ldots, V_{r}\right\}$ be the Frenet frame field on $\alpha$ and

$$
\begin{aligned}
& \lambda(s) V_{1}(s)=\left(v_{1}(s), v_{2}(s), \ldots, v_{n}(s)\right), \\
& v_{i}:[a, b] \longrightarrow R .
\end{aligned}
$$

Let the collection of curves be

$$
\begin{aligned}
& \alpha^{k}(s)=\left(\alpha_{1}(s)+k v_{1}(s), \alpha_{2}(s)+k v_{2}(s), \ldots, \alpha_{n}(s)\right. \\
& \left.\quad+k v_{n}(s)\right)
\end{aligned}
$$

for sufficiently small $k$.

For $k=0, \alpha^{0}(s)=\alpha(s)$, and $\lambda(a)=\lambda(b)=0$, we have $v_{i}(a)=v_{i}(b)=0 \quad 1 \leq i \leq n$ and $\alpha^{k}(a)=p, \alpha^{k}(b)=q$.

These results show that $\alpha^{k}$ is the curve segment from $p$ to $q$. Assume this collection $\alpha^{k}(s)=\alpha(s, k)$ for all curves. The expression for the energy of the vector field $V_{1 k}$ of $\alpha^{k}$ from $p$ to $q$ becomes $\mathscr{E}\left(V_{1 k}\right)$.

Now, let $T C_{k}$ be the tangent bundle. So we have $V_{1 k}$ : $C_{k} \rightarrow T C_{k}$, where $T C_{k}=\cup_{t \in I} T_{\alpha^{k}(t)} C_{k}, C_{k}=\alpha^{k}(I)$, and $T_{\alpha^{k}(t)} C_{k}$ denotes straight line generated $V_{1 k}$. Let $\pi: T C_{k} \rightarrow$ $C_{k}$ be the bundle projection. By using (5) we calculate the energy of $V_{1 k}$ as

$$
\begin{gathered}
\mathscr{E}\left(V_{1 k}\right)=\frac{1}{2} \int_{a}^{b} g_{S}\left(d V_{1 k}\left(V_{1 k}(\alpha(s, k))\right),\right. \\
\left.d V_{1 k}\left(V_{1 k}(\alpha(s, k))\right)\right) d s,
\end{gathered}
$$

where $d s$ is the differential arc length. From (4) we have

$$
\begin{aligned}
g_{S} & \left(d V_{1 k}\left(V_{1 k}\right), d V_{1 k}\left(V_{1 k}\right)\right) \\
= & \left\langle d \pi\left(d V_{1 k}\left(V_{1 k}\right)\right), d \pi\left(d V_{1 k}\left(V_{1 k}\right)\right)\right\rangle \\
& +\left\langle K\left(d V_{1 k}\left(V_{1 k}\right)\right), K\left(d V_{1 k}\left(V_{1 k}\right)\right)\right\rangle .
\end{aligned}
$$

Since $V_{1 k}$ is a section, we have $d(\pi) \circ d\left(V_{1 k}\right)=d\left(\pi \circ V_{1 k}\right)=$ $d\left(i d_{C_{k}}\right)=i d_{T C_{k}}$. By Proposition 4, we also have that

$$
K\left(d V_{1 k}\left(V_{1 k}\right)\right)=\nabla_{V_{1 k}} V_{1 k}=V_{1 k}^{\prime}=\frac{\partial V_{1 k}}{\partial s}
$$

giving

$$
\begin{aligned}
g_{S} & \left(d V_{1 k}\left(V_{1 k}\right), d V_{1 k}\left(V_{1 k}\right)\right) \\
& =\left\langle V_{1 k}, V_{1 k}\right\rangle+\left\langle V_{1 k}^{\prime}, V_{1 k}^{\prime}\right\rangle .
\end{aligned}
$$

Using these results in (10) we get

$$
\mathscr{E}\left(V_{1 k}\right)=\frac{1}{2} \int_{a}^{b}\left(\left\langle V_{1 k}, V_{1 k}\right\rangle+\left\langle V_{1 k}^{\prime}, V_{1 k}^{\prime}\right\rangle\right) d s,
$$

where $V_{1 k}=(1 / w(s, k))(d \alpha / \partial s)(s, k) ; w(s, k)=$ $\sqrt{\langle(d \alpha / \partial s)(s, k),(d \alpha / \partial s)(s, k)\rangle}$. 
By Definition 7, if $V_{1 k}$ is a harmonic, then $k=0$ should be a critical point of $\mathscr{E}\left(V_{1 k}\right)$. Suppose that $\left.\left(\partial \mathscr{E}\left(V_{1 k}\right) / \partial k\right)\right|_{k=0}=0$.

From (14) we obtain

$$
\begin{gathered}
\frac{\partial \mathscr{E}\left(V_{1 k}\right)}{\partial k}=\frac{\partial}{\partial k}\left[\frac{1}{2} \int_{a}^{b}\left(\left\langle V_{1 k}, V_{1 k}\right\rangle+\left\langle V_{1 k}^{\prime}, V_{1 k}^{\prime}\right\rangle\right) d s\right] \\
=\frac{1}{2} \int_{a}^{b} \frac{\partial}{\partial k}\left[\left\langle V_{1 k}, V_{1 k}\right\rangle+\left\langle\frac{\partial V_{1 k}}{\partial s}, \frac{\partial V_{1 k}}{\partial s}\right\rangle\right] d s .
\end{gathered}
$$

Since $\left\langle V_{1 k}, V_{1 k}\right\rangle=1$ we have $(\partial / \partial k)\left\langle V_{1 k}, V_{1 k}\right\rangle=0$ and we get

$$
\begin{aligned}
\frac{\partial \mathscr{E}\left(V_{1 k}\right)}{\partial k} & =\frac{1}{2} \int_{a}^{b} \frac{\partial}{\partial k}\left\langle\frac{\partial V_{1 k}}{\partial s}, \frac{\partial V_{1 k}}{\partial s}\right\rangle d s \\
& =\int_{a}^{b}\left(\left\langle\frac{\partial^{2} V_{1 k}}{\partial s \partial k}, \frac{\partial V_{1 k}}{\partial s}\right\rangle\right) d s .
\end{aligned}
$$

We can write

$$
\begin{aligned}
\frac{\partial}{\partial s}\left\langle\frac{\partial V_{1 k}}{\partial k}, \frac{\partial V_{1 k}}{\partial s}\right\rangle= & \left\langle\frac{\partial^{2} V_{1 k}}{\partial s \partial k}, \frac{\partial V_{1 k}}{\partial s}\right\rangle \\
& +\left\langle\frac{\partial V_{1 k}}{\partial k}, \frac{\partial^{2} V_{1 k}}{\partial s^{2}}\right\rangle .
\end{aligned}
$$

Thus, we can deduce

$$
\begin{aligned}
\left\langle\frac{\partial^{2} V_{1 k}}{\partial s \partial k}, \frac{\partial V_{1 k}}{\partial s}\right\rangle= & \frac{\partial}{\partial s}\left\langle\frac{\partial V_{1 k}}{\partial k}, \frac{\partial V_{1 k}}{\partial s}\right\rangle \\
& -\left\langle\frac{\partial V_{1 k}}{\partial k}, \frac{\partial^{2} V_{1 k}}{\partial s^{2}}\right\rangle .
\end{aligned}
$$

Substituting (18) in (16), for $k=0$,

$$
\begin{aligned}
& \left.\frac{\partial \mathscr{E}\left(V_{1 k}\right)}{\partial k}\right|_{k=0}=\int_{a}^{b}\left[\frac{\partial}{\partial s}\left\langle\frac{\partial V_{1 k}}{\partial k}(s, 0), \frac{\partial V_{1 k}}{\partial s}(s, 0)\right\rangle\right. \\
& \left.-\left\langle\frac{\partial V_{1 k}}{\partial k}(s, 0), \frac{\partial^{2} V_{1 k}}{\partial s^{2}}(s, 0)\right\rangle\right] d s, \\
& \left.\frac{\partial \mathscr{E}\left(V_{1 k}\right)}{\partial k}\right|_{k=0}=\left.\left\langle\frac{\partial V_{1 k}}{\partial k}(s, 0), \frac{\partial V_{1 k}}{\partial s}(s, 0)\right\rangle\right|_{a} ^{b} \\
& -\int_{a}^{b}\left\langle\frac{\partial V_{1 k}}{\partial k}(s, 0), \frac{\partial^{2} V_{1 k}}{\partial s^{2}}(s, 0)\right\rangle d s .
\end{aligned}
$$

From (8) and (9), we obtain

$$
\begin{aligned}
& \frac{d \alpha}{\partial k}(s, k)=\lambda(s) V_{1}(s), \\
& \frac{d \alpha}{\partial s}(s, 0)=\alpha^{\prime}(s)=V_{1}(s, 0) .
\end{aligned}
$$

Now we calculate the partial derivatives of (22) with respect to $s$ and $k$; using Frenet formulas, we get

$$
\begin{aligned}
\frac{\partial V_{1 k}}{\partial s}(s, 0) & =\frac{\partial^{2} \alpha}{\partial s^{2}}(s, 0)=\alpha^{\prime \prime}(s)=V_{1 k}^{\prime} \\
& =k_{1}(s) V_{2}(s), \\
\frac{\partial V_{1 k}}{\partial k}(s, k) & =\frac{\partial^{2} \alpha}{\partial s \partial k}(s, k)=\frac{\partial^{2} \alpha}{\partial k \partial s}(s, k) .
\end{aligned}
$$

From (21), we have

$$
\begin{aligned}
\left.\frac{\partial V_{1 k}}{\partial k}(s, k)\right|_{k=0} & =\frac{\partial V_{1 k}}{\partial k}(s, 0) \\
& =\lambda^{\prime}(s) V_{1}(s)+\lambda(s) k_{1}(s) V_{2}(s) .
\end{aligned}
$$

It follows from (23) and (25) that

$$
\left\langle\frac{\partial V_{1 k}}{\partial k}(s, 0), \frac{\partial V_{1 k}}{\partial s}(s, 0)\right\rangle=\lambda(s) k_{1}^{2}(s) .
$$

Considering the candidate function $\lambda(a)=\lambda(b)=0$, we get

$$
\begin{aligned}
& \left.\left\langle\frac{\partial V_{1 k}}{\partial k}(s, 0), \frac{\partial V_{1 k}}{\partial s}(s, 0)\right\rangle\right|_{a} ^{b} \\
& =\lambda(b) k_{1}^{2}(b)-\lambda(a) k_{1}^{2}(a)=0 .
\end{aligned}
$$

From (23), we get

$$
\begin{aligned}
\frac{\partial^{2} V_{1 k}}{\partial s^{2}}(s, 0)= & -k_{1}^{2}(s) V_{1}(s)+k_{1}^{\prime}(s) V_{2}(s) \\
& +k_{1}(s) k_{2}(s) V_{3}(s) .
\end{aligned}
$$

Therefore, (25) and (28) give

$$
\begin{aligned}
& \left\langle\frac{\partial V_{1 k}}{\partial k}(s, 0), \frac{\partial^{2} V_{1 k}}{\partial s^{2}}(s, 0)\right\rangle \\
& =\left[-\lambda(s) k_{1}^{2}(s)\right]^{\prime}+3 \lambda(s) k_{1}(s) k_{1}^{\prime}(s) .
\end{aligned}
$$

Substituting (27) and (29) in (20) yields

$$
\begin{aligned}
& \left.\frac{\partial \mathscr{E}\left(V_{1 k}\right)}{\partial k}\right|_{k=0} \\
& =-\int_{a}^{b}\left(\left[-\lambda(s) k_{1}^{2}(s)\right]^{\prime}+3 \lambda(s) k_{1}(s) k_{1}^{\prime}(s)\right) d s, \\
& \left.\frac{\partial \mathscr{E}\left(V_{1 k}\right)}{\partial k}\right|_{k=0} \\
& =\left.\left[-\lambda(s) k_{1}^{2}(s)\right]\right|_{a} ^{b}-3 \int_{a}^{b} \lambda(s) k_{1}(s) k_{1}^{\prime}(s) d s=0 .
\end{aligned}
$$

Since $\lambda(a)=\lambda(b)=0$, it gives $\left.\left[-\lambda(s) k_{1}^{2}(s)\right]\right|_{a} ^{b}=0$ and

$$
\left.\frac{\partial \mathscr{E}\left(V_{1 k}\right)}{\partial k}\right|_{k=0}=-3 \int_{a}^{b} \lambda(s) k_{1}(s) k_{1}^{\prime}(s) d s=0
$$

This completes the proof of the theorem. Also, it is trivial that geodesics and curves with constant curvature satisfy the theorem. 


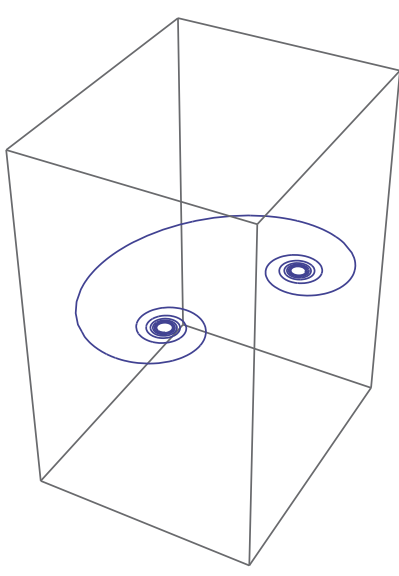

(a)

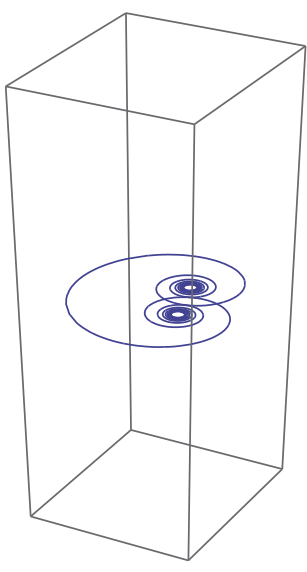

(b)

FiguRE 1: (a) $\kappa_{1}(s)=5 s^{2}+3, \kappa_{2}(s)=0$ (b) $\kappa_{1}(s)=s^{2}+1, \kappa_{2}(s)=0$.

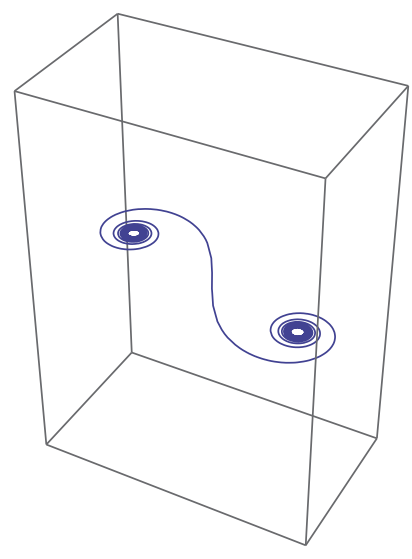

(a)

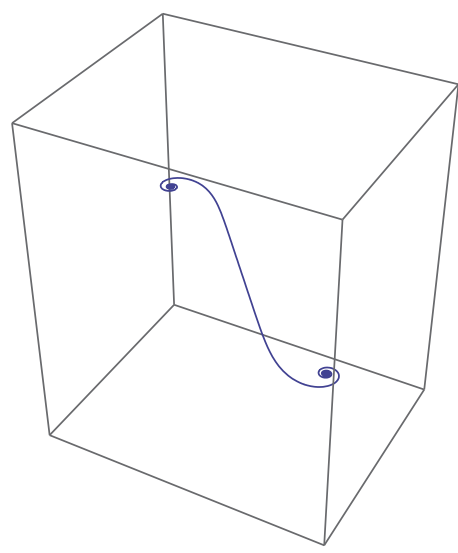

(b)

Figure 2: (a) $\kappa_{1}(s)=5 s, \kappa_{2}(s)=0$, (b) $\kappa_{1}(s)=5 s^{5}, \kappa_{2}(s)=0$.

We give an example that provides the condition (7) in the theorem below. Using different curvatures, we illustrate the example with Figures 1 and 2.

Example 9. Let $\alpha: I \rightarrow R^{3},[-1,1] \subset I, \alpha(-1)=p, \alpha(1)=q$. If we can choose $\lambda:[-1,1] \rightarrow R, \lambda(s)=1-s^{2}, \lambda(-1)=0$, $\lambda(1)=0$, and $\lambda(s) \neq 0$ for all $s \in(-1,1)$ then the curves which have $\kappa(s)=c s^{2 n}+d, \kappa(s)=c s^{2 n+1}(c, d \in R$ and $n \in N)$ provide condition (7).

Figures 1 and 2 are shown in 3-dimensional space as sample to $n$-dimensional space.

\section{Conflicts of Interest}

The authors declare that they have no conflicts of interest.

\section{References}

[1] E. Kreyszig, Differential geometry, Dover Publications, 1991.
[2] H. Gluck and W. Ziller, "On the volume of a unit vector field on the three-sphere," Commentarii Mathematici Helvetici, vol. 61, no. 2, pp. 177-192, 1986.

[3] D. L. Johnson, "Volumes of flows," Proceedings of the American Mathematical Society, vol. 104, no. 3, pp. 923-932, 1988.

[4] A. Higuchi, B. S. Kay, and M. C. M. Wood, "The energy of unit vector fields on the 3-sphere," Journal of Geometry and Physics, vol. 37, no. 1-2, pp. 137-155, 2001.

[5] C. M. Wood, "On the energy of a unit vector field," Geometriae Dedicata, vol. 64, no. 3, pp. 319-330, 1997.

[6] A. Altın, "On the energy and the pseudo angle of a frenet vectors fields in $R_{v}{ }^{n}$," Ukrainian Mathematical Journal, vol. 63, no. 6, pp. 833-839, 2011.

[7] B. O’Neill, Elementary Differential Geometry, Academic Press Inc, 1966.

[8] P. M. Chacón, A. M. Naveira, and J. M. Weston, "On the energy of distributions, with application to the quaternionic Hopf fibrations," Monatshefte fûr Mathematik, vol. 133, no. 4, pp. 281-294, 2001. 
[9] P. M. Chacón and A. M. Naveira, "Corrected energy of distributions on Riemannian manifolds," Osaka Journal of Mathematics, vol. 41, no. 1, pp. 97-105, 2004.

[10] P. Baird and J. C. Wood, Harmonic Morphisms between Riemannian Manifolds, Clarendos Press, Oxford, 2003. 


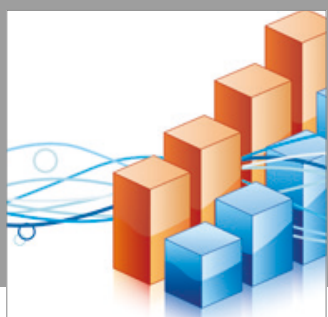

Advances in

Operations Research

vatersals

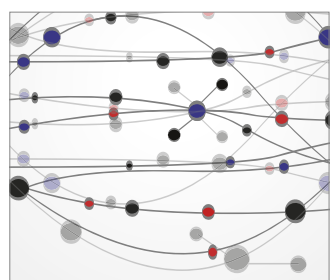

\section{The Scientific} World Journal
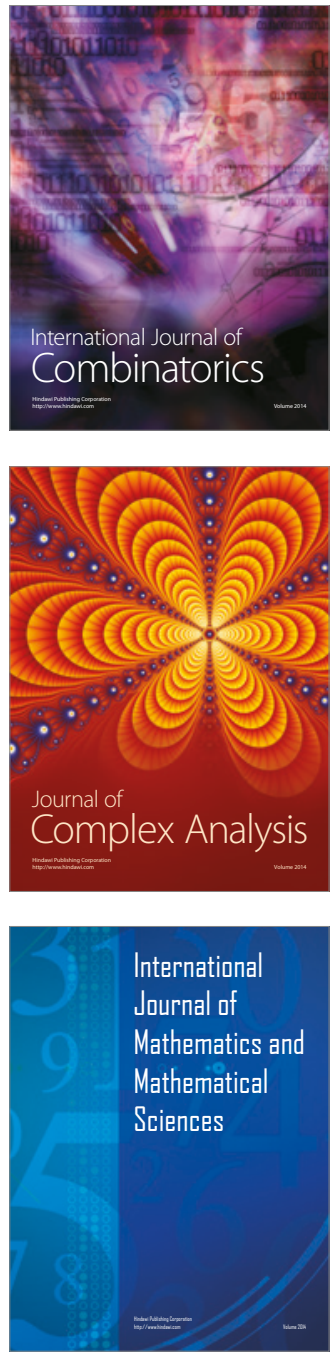
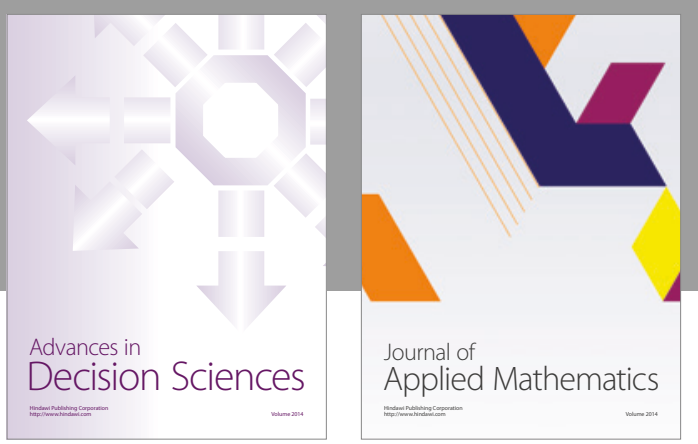

Algebra

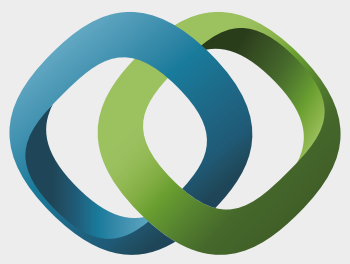

\section{Hindawi}

Submit your manuscripts at

https://www.hindawi.com
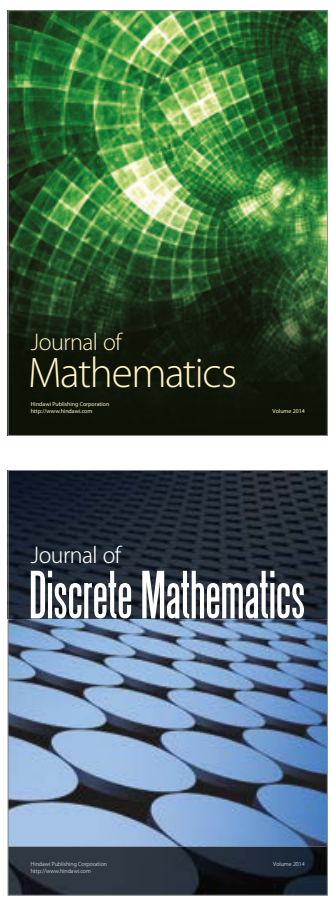

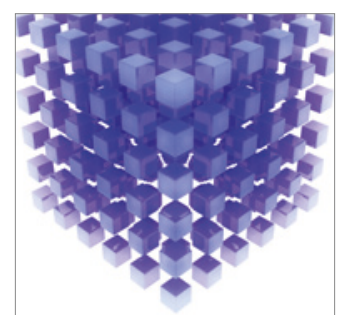

Mathematical Problems in Engineering
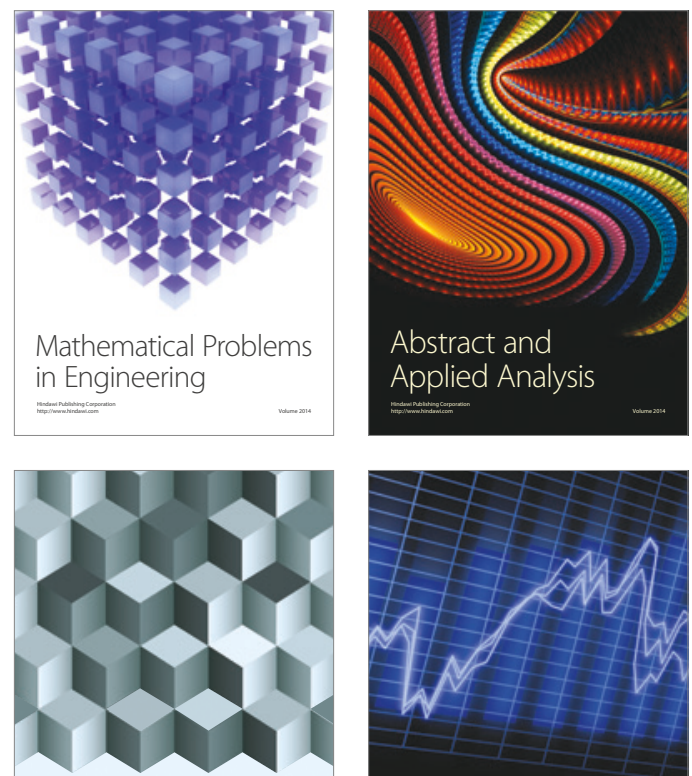

Journal of

Function Spaces

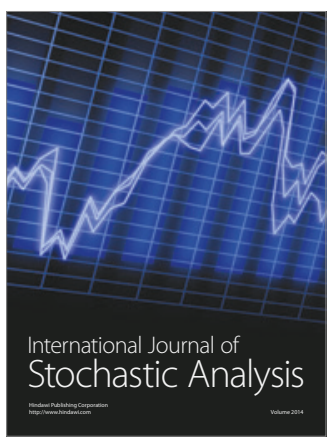

Probability and Statistics
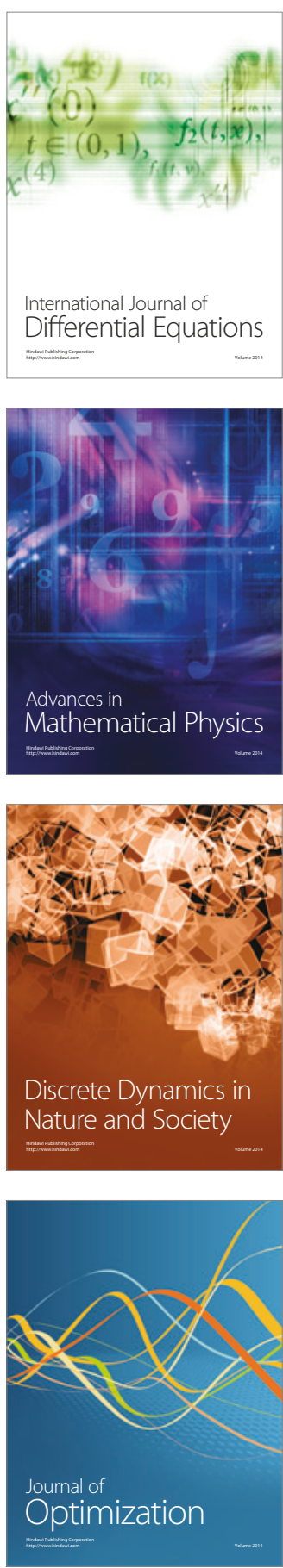